\title{
Assessing the Present-Day Impact Flux to the Lunar Surface Via Impact Flash Monitoring and Its Implications for Sustained Lunar Exploration
}

\author{
A White Paper for the National Academies \\ Planetary Science and Astrobiology Decadal Survey
}

15 July 2020

Joshua T.S. Cahill, Johns Hopkins University Applied Physics Laboratory

(e-mail: Joshua.Cahill@jhuapl.edu; phone: 808-220-9389)

Emerson J. Speyerer, Arizona State University

(e-mail: espeyerer@ser.asu.edu)

Contributors:

Debra Hurwitz Needham, NASA Marshall Space Flight Center

Renee Weber, NASA Marshall Space Flight Center

Ingrid Daubar, Brown University

Emily Costello, University of Hawai'i-Manoa

Daniel Moriarty, NASA Goddard Space Flight Center

Robert Suggs, NASA Marshall Space Flight Center

Raven Larson, University of Colorado Boulder

Ryan Watkins, Planetary Science Institute

Angela Stickle, Johns Hopkins University Applied Physics Laboratory

Benjamin Greenhagen, Johns Hopkins University Applied Physics Laboratory

Co-Signers:

Heather Meyer, Johns Hopkins University Applied Physics Laboratory

Carolyn Ernst, Johns Hopkins University Applied Physics Laboratory

Samuel Lawrence, NASA Lyndon B. Johnson Space Center

Blair DeWitt, Lunar Station Corp.

Rebecca Ghent, Planetary Science Institute 
One-Sentence Summary \& Recommendation. Near-term, robust investigations of presentday impact flash and flux observations of the lunar surface would greatly benefit efforts to answer a number of important science questions, and more importantly, help quantify hazards that will be encountered by sustained robotic, human, and ultimately habitation endeavors on and below the lunar surface.

Box 1- Priority science and exploration questions answered by impact flash and flux investigations and relative alignment with :SCEM \& "LER documents.

- How can we utilize the Moon as a natural laboratory to study impact flashes and the resulting craters to better inform laboratory experiments, numerical models, the cratering record, and ultimately the hazard they pose to sustained exploration? (SCEM 1c, 1e, 6d; LER Sci-A-7, B1)

- What is the present-day lunar impact flux and its range of meteoritic infall size? (SCEM 1c, LER Sci-B-1)

- What does the present-day lunar impact flux imply about the impact flux at Earth and other bodies in the Solar System; particularly those of interest for human exploration (e.g., Mars)? (LER Sci-B-1)

- What is the present-day spatial distribution of lunar bombardment? Does it vary temporally? Is the impact energy/flux higher on the Western (leading) or Eastern (trailing) hemisphere of the Moon relative to current models? Do the lunar poles incur the lowest impact flux? (SCEM $6 \mathrm{c}, 6 \mathrm{~d})$

- What are the geologic effects of present-day impact cratering on the Moon (e.g., resurfacing/gardening, seismic shaking, triggering of landslides, and range of influence)? (SCEM 6c, 6d, 7c; LER Sci-A-7, D-22, FF-C-1)

- What volatiles are detectable in lunar impact plumes? If present, how are they redistributed, re-consolidated, and what percentage is lost? (SCEM 6c, 7c)

- What can fresh exposures of new impact craters tell us about the physical nature of the lunar surface (exposure of volatiles, space weathering, layering/strength transitions...)? (SCEM 6c, 6d, and 7b, c; LER Sci-A-7)

*Scientific Context for Exploration of the Moon; "Lunar Exploration Roadmap

\section{Introduction}

The sudden flashes of light generated by meteoroid impacts of the lunar surface are evidence that the lunar surface is not static, but extraordinarily more dynamic than appreciated (Figure 1). Further, increasing evidence suggests the present rate of bombardment is greater than anticipated and the actual and modeled rates are less constrained and understood than necessary for informed, sustained human exploration. Additional first hand, and unobscured, observations are necessary to formulate a hazard mitigation, or even a defensive strategy, during exploration of the lunar surface. To both the science and exploration communities' benefit, any impact flash, plume, and resulting crater provide an opportunity to assess many priority lunar and Solar System science and exploration questions (Box 1).

It may come as a surprise that capturing an impact flash event on video unambiguously was elusive until only recently, at the turn of the century, during the Leonid storm of 1999 [1-4] and the 2001 Leonids [5-6]. Each impact flash puts on a catastrophic yet fleeting show (usually nanoto milliseconds), but reveals quite a bit of immediate information (Figure 1) regarding location, temperature, size, and some indication of frequency. In fact, the luminous energy of an impact flash yields valuable information to constrain the size ranges of meteoroids difficult to observe with other means [7]. Recently, it has become more apparent that the culmination of impact bombardment from microscopic to kilometer-sized objects over time, or the impact flux, may be more poorly constrained for the Moon and the rest of the Solar System than previously appreciated [8]. This is because there is only so much we can observe and detect from Earth since our atmosphere obscures much of the information from smaller impactors, and our interpretation of orbital data rests on historical and incomplete temporal observations. What we can observe from 
Earth could be enhanced through improved instrumentation and coordination of interested investigators; however, even then there are limits to what can be detected. What will ultimately be needed is an impact flash monitoring system, utilizing a combination of Earth-based, lunar surfacebased, and orbital observations.

In this White Paper we outline how the study of lunar impact flash and flux could yield important information for science and practical information for human exploration and habitation of the Moon. Present day impact bombardment (primary and secondary, large to microscopic) and its flux is a potential hazard for planetary exploration and must be sufficiently assessed (Ghent and Zellner [9] stress the need for additional methods and opportunities for measurement as well). Improved collection of this knowledge is crucial as robotic and, more importantly, human exploration on the lunar surface is planned to re-start and accelerate with the advent of NASA's Artemis Program. As humans return to the Moon to stay, methods of hazard assessment/monitoring for planning, adaptation, and mitigation (when necessary) should be formulated and continually refined.

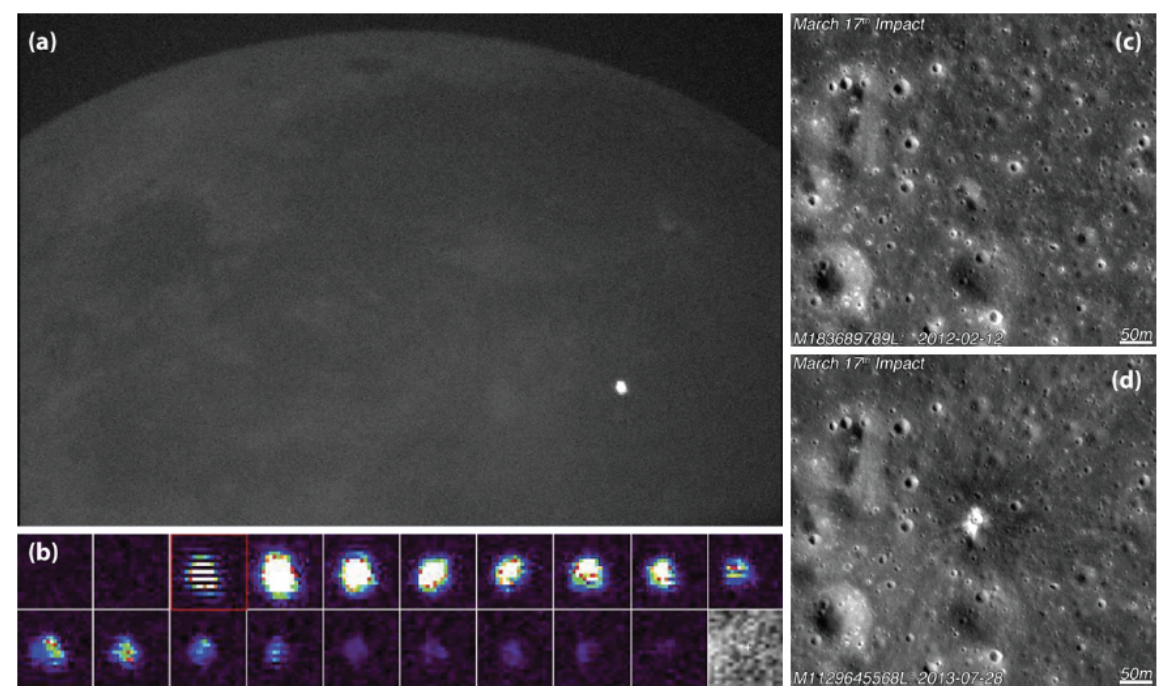

Present State of Knowledge
Figure 1. From Suggs et al. (2014) - Lunar impact flash observations from March 17, 2013. Panel (a) is the full field-of-view of the brightest frame collected at NASA MSFC. Panel (b) is the sequence of successive $1 / 30$ s video frames zoomed in. Panel (c) is the before and $(d)$ the after image of the $18 m$ crater impact site captured by the Lunar Reconnaissance Orbiter Camera.

Past and Ongoing Investigations - Eyewitness accounts of so-called lightning and starlight on the eclipsed hemisphere of the Moon, which may refer to optical flashes from impact events, date back to at least 1178 [10-11]. In 1956, the first such impact event may have been photographed through a telescope [12]. With the advancement of digital video recording devices, hundreds of impact flashes captured by observatories around the globe provide insight into the contemporary impact rate on the Moon's nearside [3,7,13]. From these flash observations, researchers estimate the impact energy and derive crater diameter. By tying flash events to meteor streams, researchers can infer additional impact characteristics such as the projectile density, velocity, and direction.

With the launch of the Lunar Reconnaissance Orbiter (LRO) in 2009, meter-scale observations acquired by the Lunar Reconnaissance Orbiter Camera (LROC) Narrow Angle Camera (NAC) provide the means to identify and characterize new impact events. Daubar and colleagues [14] identified five new impact craters by manually comparing Apollo Panoramic Camera and NAC images covering $0.015 \%$ of the Moon. The increased spatial and temporal coverage obtained during the LRO extended mission led to the discovery of two new 18- and 34-m impact craters whose flashes were observed using Earth-based impact flash monitors [7, 13, 15]. In addition to locating the impact site, the NAC images provided accurate measurements of crater diameters; for example, the $34 \mathrm{~m}$ impact crater identified using a recorded impact flash on 11 September 2013 had a predicted diameter of 47-56 m [13]. Building upon these discoveries and the growing imaging time-base, Speyerer and co-authors [8] scanned 14,092 NAC temporal pairs (before and 
after NAC images with similar lighting) and found 222 newly formed impact craters. As part of the study, they found that the contemporary impact rate is potentially $33 \%$ higher than predicted by the Neukum crater production function [16]. Continued surveys of temporal observations since the initial investigation has led to the discovery of over 500 newly formed impact craters detectable with current observations resources just within the course of the LRO mission ( 11 years).

High-resolution, temporal observations have also led to the identification of distal scattered ejecta, commonly referred to as splotches, around many of the newly formed large $(>15 \mathrm{~m})$ impact craters. Robinson et al. [15] identified 248 new splotches that formed on the surface during the impact event. The herringbone shape of some of the new splotches indicated an emplacement direction pointing back to the primary impact crater, supporting the hypothesis that many are secondary formations. Temporal pair surveys [8, 17] have helped identify over 120,000 new splotches on the lunar surface that have formed in the last decade. These secondary debris effects are of interest to exploration because they dramatically amplify the hazards to resources as they disperse an apron of projectiles laterally, amplifying the effects of the initial impact. Secondary impacts, although less energetic than primaries, pose a physical hazard to active exploration because they fall so frequently. With a sustained presence on the Moon, it is far more likely astronauts and/or infrastructure would be affected by a secondary than by a primary impact though both are equally bad.

Even our current orbital asset around the Moon, LRO, has not been spared from bombardment. In late 2011 to early 2012, the flight operations team observed a drop in the open-circuit voltage on the LRO solar array. The magnitude was consistent with the loss of a solar cell which may be attributed to a potential meteoroid strike. Then again, in December 2014, a small meteoroid struck the left NAC camera while it was acquiring an observation, causing a high frequency distortion across the image [18]. Separately, the Lunar Dust Experiment (LDEX) instrument on the Lunar Atmosphere and Dust Environment Explorer (LADEE) mission has also recorded more dynamic lunar activity than initially appreciated. The LDEX instrument helps characterize and measure the physical properties of particles impacting the detector. During the LADEE mission, the LDEX team tied spikes in impact detections to meteor showers, then also showed the nearly-continuous hits were attributed to the spacecraft flying through ejecta clouds that were the result of impact events on the lunar surface [19].

Present Limitations to Monitoring Impact Flash \& Flux - Present day observations of lunar impact flashes are limited to several observatories and research centers around the world. While the studies being conducted are robust, there are many limitations that hinder flash observations. First, observations can only be made of the nearside and typically only when the Moon is illuminated $10-45 \%$ due to stray light from the illuminated surface or the Moon's proximity to the Sun in the sky [20]. To further reduce the observation time, local weather can affect the ability to collect observations and can ultimately bias seasonal measurements (i.e., spring storms and/or cloudy winters may reduce flash counts during those months). These observations are also made through the atmosphere, which can distort flash observations that add uncertainty to flash magnitude estimates. Finally, the air-traffic and orbital assets around the Earth can sometimes cause glints that appear like impact flashes and thus require multiple observations from separate locations to confirm a single flash event.

The number of impacts a single observatory can capture is limited by the size of the telescope and observation conditions. To detect smaller impacts from Earth, some observatories are moving to larger telescopes than the 14-inch and 0.5-m telescopes used at NASA's Marshall Space Flight Center (MSFC). By comparison, the 1.2-m telescope in Greece [20] collects over 10x as much light as the standard 14-inch telescope at MSFC and can detect some of the dimmest impact flashes (12th magnitude in $\mathrm{R}$ filter). These magnitudes correspond to new craters down to 1.2 meters in diameter.

Observations from the Earth-Moon Lagrange Points and/or lunar orbit would provide an unobstructed view of the lunar surface to not only detect flashes, but possibly characterize the 
impact events (i.e., ejecta dispersal, plume characteristics and composition, and volatile content). Models based upon Neukum [16] estimate a $\geq 10 \mathrm{~m}$ crater forming on the surface every $\sim 2.5$ days. However, recent observations have suggested these models may underestimate the flux by $33 \%$ [17]. Furthermore, recent Earth based observations by Liakos et al. [20] have detected a baseline (i.e., non-meteor shower) rate equivalent to $\sim 8$ meteoroid hits per hour corresponding to craters down to almost 1 meter in diameter. It is probable that an observatory at L1 or L2 would have the capability to detect flashes from meteoroid impacts of this scale and smaller, adding the ability to further constrain the impact flux. However, crater diameters then must be determined and require subsequent high-resolution orbital observations (e.g., LROC) to confirm the final crater size. An orbital high-resolution camera (LROC or next generation imager) could then subsequently confirm the impact events and provide accurate diameter measurements, which can be used to further constrain impact models and better characterize the initial bolide. Further, characterization of the impact site through examination of temporal pairs could also constrain the magnitude and distribution of distal scattered ejecta or splotches. While these secondaries are not as energetic, they pose a physical hazard to active exploration due to their numbers and wide spatial distribution relative to the initial impactor(s).

\section{Relevance to Multiple Community Objectives}

The importance of impact cratering, including the flux, has been extensively highlighted in both the Scientific Context for Exploration of the Moon (Science Goal 1c, 1e, 6c, 6d, 7b, and 7c) and the Lunar Exploration Roadmap (Sci-A-7, B-1, D-22, FF-C-1). Impact flux was also noted in the previous decadal survey multiple times [21]. However, when noted, this important science question's relevance has primarily been referred to as a historical rather than present-day Solar System science question. The fact is that more precise knowledge of present-day impact flux conditions will better constrain our knowledge of our Moon's past (further discussed in [9]), and will be a crucial piece of information to constrain the Moon's internal structure and seismicity via the Lunar Geophysical Network (LGN; [22]). In fact, impact flash and flux monitoring are now requirements of the LGN mission concept [22]. Knowledge of each impact's magnitude, positioning, and timing will help us discern seismic events generated at the surface from those produced in the subsurface further refining our understanding of the Moon's interior structure and seismicity.

Studies of impact plumes will also be informative for studies of volatiles, as was undertaken by LCROSS observations of the spacecraft impact within Cabeus crater [23]. Finally, and perhaps most importantly, as NASA's Artemis Program endeavors to return to the Moon's surface with both government and commercial spacecraft, a number of unique challenges present themselves. The Artemis effort entails sustained human and robotic activities on the lunar surface. This fact presents a unique set of potential hazards to assess, one of which is the potential for bombardment by primary and/or secondary impactors, both large and microscopic. In contrast to the Earth's atmosphere, the tenuous lunar exosphere provides negligible shielding, subjecting humans and their robotic tools and habitats to the fullest extent of bombardment.

\section{Proposed Orbital Configuration for Lunar Impact Flash Observation \& Flux Assessments}

At present, there is no domestic lunar impact flash mission slated by NASA for conceptual development or launch. With humans approaching a return to the Moon, unknowns regarding the lunar impact flux environment present a probable exploration knowledge gap that should be more widely and readily recognized by the scientific and exploration community. Domestically, we have some potential options. Lunar Gateway may offer payload accommodation options to leverage in terms of either impact flash monitoring or subsequent characterization of the crater and its secondary ejecta. However, that infrastructure is still under development and its details are varying fluidly. A more beneficial option would be a dedicated orbital mission coordinated with Earth-based observations for impact flash and flux monitoring.

An orbiter at the second Earth-Moon Lagrange Point (L2) would provide the minimal infrastructure required to get near global impact flash coverage. At L2, impact flash events 
normally undetectable on the lunar far side could be observed for study. This would create a small network in which Earth observers collect observations from the nearside of the Moon and the L2 orbiter collects impact flash observations from the farside of the Moon. If the orbiter was also placed in a vertical halo orbit at L2 (Figure 2), it would ensure better views of the North and South Poles at different times during its orbit. Of great importance, the orbiter would also function as a communications satellite for human and robotic activities on the lunar farside and in polar regions (a similar dual-purpose communications and impact flash/flux satellite, IRIS, was previously proposed in the MoonRise concept; Clegg et al., 2011).

The configuration above would be a good first step; however, we have already briefly described the limitations of Earth-based impact flash observations due to its atmosphere and weather. We recommend spacecraft at both the L2 and L1 Earth-Moon Lagrange points, which would provide more comprehensive coverage and superior sensitivity for science and hazard awareness. These spacecraft would be configured to be closest to opposite poles for the best total coverage of the Moon for impact flash events, and the most capable configuration for communications (Figure 2). This would improve coverage of the nearside polar perspectives and improve sensitivity for impact flash detections free of atmospheric interference. Additional Lagrange point missions at L4 and/or L5 for entirely different purposes could also enhance the impact flash observation network if they were outfitted with impact flash instrument payloads. But, without further study, we currently do not think this would be required to drastically improve the current state of impact flash monitoring for both science and exploration purposes.

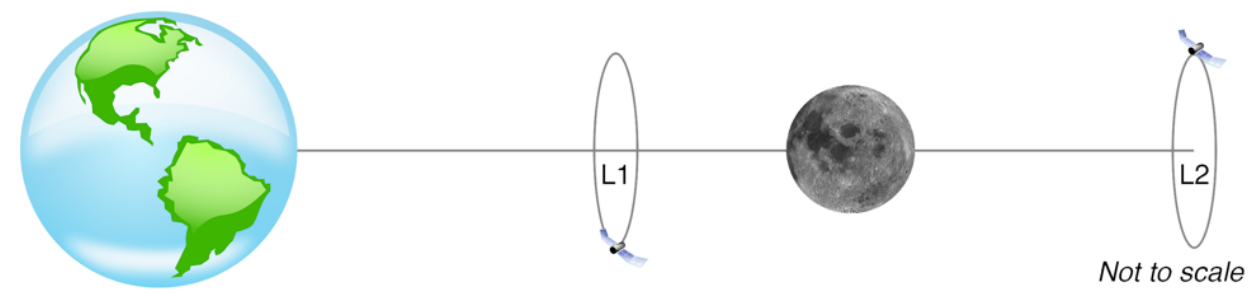

Figure 2. Lagrange point observation 'perches' for impact flash and cis-lunar communications.

Once an impact flash is detected, a spacecraft in a closer (e.g., 50 to $100 \mathrm{~km}$ ) orbit to the Moon would provide post-impact reconnaissance of the crater and debris field. We expect this may be an orbiter outfitted like a Lunar Reconnaissance Orbiter 2 (LRO2), which is outlined in a mission White Paper by Glotch [24], or with resolution capabilities that improve upon LROC's NAC. The objective would be to better establish location, characteristics of the impact relative to the energy detected from the flash, and the area it influenced by its proximal, distal, and scattered ejecta.

\section{Recommended Orbital Instrument Payloads}

Notional payloads that could be utilized to study impact flash individually or in a highly complementary manner are listed below. This should not be considered a comprehensive list, but the near minimum technology necessary to achieve the needed observations to begin answering the science and exploration questions posed earlier (Box 1).

\section{Lagrange Point Orbital Payloads:}

High Speed Multiband Radiometer - A radiometer with enough sensitivity to detect flashes and profile temporal temperatures for both day and night illumination is necessary [25]. The radiometer will continuously monitor the full observable lunar disk and the radiometric signal will be continually buffered. Data will only be saved when an event occurs. During flash events, the rapid rise in intensity seen by the radiometer will trigger the archiving of other instrument's observations.

High Speed Visible Imager - Cameras utilized for laboratory measurements of impact flash generally run between $500,000-10^{\wedge} 6$ frames/second; but lab impact flash events usually last nano- to milliseconds. However, MSFC facilities have demonstrated effectiveness during lunar impact flash surveys with cameras collecting 30-50 frames/second. This usually gives at least a 
couple image frames of each flash event (e.g., Figure 1). A dedicated impact flash camera should build upon this, and options for 200+ frames/second are available (e.g., Malin cameras for DragonFly). But, the engineering trade-off is data storage. A $2 \mathrm{k} \times 2 \mathrm{k}$ array running at 100 frames/second with 12 bits/pixel results in $~ 5$ Gbits/second. For perspective, that is roughly $50 \%$ greater data volume collected than Europa Clipper's Europa Imaging System (EIS). The proposed camera would also continually fill a buffer of images, but images would only begin to be archived when the radiometer is triggered by a flash event.

Hyperspectral Imager/Video - Such instruments will be valuable for observing the initial impact and analyzing plume dynamics, dispersal, and composition (i.e., mineral, chemical, and volatile abundances). This instrument would need a high enough frame rate to be comparable to the high-speed imager at some level. Multiple studies demonstrated how hyperspectral instrumentation could also be valuable for plume and other chemical species studies during the LCROSS impact of Cabeus crater and should be considered for any 'impact witness mission'. For example, LRO's LAMP, a far-ultraviolet imaging spectrograph, showed it was possible to detect the LCROSS impact plume with $\sim$ millisecond time resolutions [26]. Similar instruments would be extremely useful for identifying chemical species and any volatiles in lunar impact plumes [27]. Hyperspectral video capabilities are also in use for present day tracking purposes and should prove useful for tracking plume materials and secondary ballistic debris.

\section{Post-Impact Analysis Orbiter (e.g., LRO2):}

Multispectral Stereo Imager - A high-resolution multispectral stereo imager $(\leq 0.5 \mathrm{~m} / \mathrm{pix})$ enables detailed studies of the crater morphology, ray formation, mixing, and space weathering state [24]. A digital domain time-delay-integration (dTDI) push-broom camera with native stereo capabilities enables capture of multiple narrow color bands and a broadband filter. The stereo would enable accurate crater depth/diameter calculations as well as collect a robust photometric dataset. These observations can also be directly compared to past LROC observations to detect other new impact craters as well as analyze the production of distal secondaries and splotches.

\section{Improving \& Coordinating Earth Based Observation Impact Flash Events}

To improve Earth-based observation of impact flash events, a network of coordinated observations could be organized to provide complete observation of the Moon during the crescent $(10 \%)$ to quarter $(45 \%)$ phases. To detect the largest impact events, large telescopes are not required to effectively capture and characterize the flash. Many universities have some sort of telescopic facility that would be suitable to join a flash monitoring campaign. The minimum instrumentation needed are: 1) telescope ( $\geq 10$ inch), 2) a high-speed camera that collects $>20$ frames/second, 3) data storage facilities, and 4) suitable tracking software to keep the telescope field of view in the same position on the Moon within each framed observation. Even though calibrated detectors are preferable, modern consumer-grade DSLR cameras are common these days and could enable engagement of a larger scientific community to help close observation gaps in the current data set rapidly. Current software tools (i.e., MSFC's LunarScan or MIDAS) enable users to process and systematically analyze video observations to detect flash events. To improve geolocation of flash events, popular visualization tools (i.e., LROC Quickmap) could be retrofitted to provide synthetic Earth views of the Moon that account for observation time and telescope location for improved image registration. Additional flash tracking tools and databases would enable multiple institution's research groups to collaborate to improve impact flash statistics. We stress that while this endeavor could help improve current knowledge, it could not substitute for the orbital observation capabilities proposed here.

\section{Lunar Surface Based Impact Flux Observations}

As verified in laboratory experimentation, impact flashes occur even at the microscopic level. However, even with a robust orbital survey of impact flashes, some smaller meteoroids may evade detection. But, accounting for impactors of this size is crucial for a full understanding of the lunar impact flux [9] and all the hazards they present. A methodology to collect this information may be 
filled with observation equipment on robotic assets and habitats. For example, the flux and characteristics of the smallest impactors (below the sensitivity of orbital and seismic network sensors) can be evaluated through particle capture. A concept utilizing aerogel panes to capture these particles [28], or something similar, ideally would be deployed routinely by a CLPS lander or Artemis astronaut for a period of time at diverse locations around the Moon, then returned (and possibly replaced) for Earth analysis in terrestrial laboratories. Based upon data from various lunar orbiters, a $1 \mathrm{~m}^{2}$ aerogel pane on the lunar surface would be subject to $\sim 3$ impacts per year of $\mu \mathrm{g}$ mass micrometeorites [29]. This number sounds inconsequential, but is expected to increase exponentially with decreasing impactor mass. As a result, bombardment of robotic assets and habitats by micrometeorites should not be underestimated in the long term as such exposure is likely not inconsequential to their functionality nor longevity.

\section{Utilization of Planetary Defense Infrastructure for Lunar Activities}

Once humans are on the Moon persistently, planetary defense objectives may begin to expand. Following the Double Asteroid Redirection Test (DART), the next planetary defense objective consists of an infrared near-Earth object (NEO) survey telescope known as the NEO Surveillance Mission (NEOSM; [30-31]). If such a mission would advance, an opportunity may exist for such a survey's data to be utilized for the secondary purpose of screening meteoroids of detectable scales that will impact the lunar surface. This could advance lunar surface exploration planning by limiting or sheltering robotic resources on the lunar surface, evacuating humans prior to impact, and planning coordinated observations of a meteoroid prior to, during (impact flash observations), and after the impact. Such information from the natural environment could be extraordinarily informative regarding the entire impact process (projectile, target, and at scale).

\section{Findings and Recommendations}

Lunar impact flash monitoring not only elucidates information about the present-day impact flux, but also provides key information and observations needed to better understand the impact process itself, which is a fundamental aspect to how planetary bodies are formed and shaped. Using the Moon as a witness plate, we can capture larger impact events than can be detected from Earthbased observatories. With an L2 and possible L1 monitor, along with coordinated Earth-based observations, we can increase our monitoring coverage and improve our impact characterizations and statistics dramatically. The recommended instrumentation, as outlined above, will further characterize and improve our insight and understanding of the cratering process which will help not only improve our scientific knowledge of this fundamental process, but also the potential and persistent risks to sustained surface operations by human and robotic explorers.

\section{References}

[1] Bellot Rubio et al. (2000) Earth, Moon, Planets 82-83, 575-598. [2] Dunham et al., (2000) LPSC, Abs\# 1547. [3] Ortiz et al., (2000) Nature 405, 921-923. [4] Yanagisawa and Kisaichi (2002) Icarus 159, 31-38. [5] Cudnik et al. (2002) LPSC, Abs\# 1329. [6] Ortiz et al. (2002) Astrophys. J. 576, 567-573. [7] Suggs et al. (2014) Icarus 238, 23-36. [8] Speyerer et al. (2016) Nature 538, 215-218. [9] Ghent and Zellner (2020) Decadal White Paper. [10] Cameron (1972) Icarus 16, 339-387. [11] Hartung (1976) Meteoritics 11, 187-194. [12] Stuart (1956) Stroll. Astron. 10, 42-43. [13] Madiedo et al. (2014) Mon. Notices of the Royal Astro. Soc. 439, 23642369. [14] Daubar et al. (2011) LPSC, Abs\# 2232. [15] Robinson et al. (2015) Icarus 252, 229235. [16] Neukum et al. (2001) Space Sci. Rev. 96, 55-86. [17] Speyerer et al. (2019) GSA, Abs\# 340819. [18] Robinson (2017) http://lroc.sese.asu.edu/posts/973. [19] Szalay and Horanyi (2016) Icarus 275, 221-231. [20] Liakos et al. (2020) Astronomy \& Astrophysics 633, A112. [21] NRC (2011) V\&V Decadal Survey. [22] Weber (2020) Decadal White Paper. [23] Colaprete et al. (2010) Science 330, 463-468. [24] Glotch (2020) Decadal White Paper. [25] Stickle et al. (2018) Deep Space Gateway Sci. Wkshp., Abs\# 2063. [26] Gladstone et al. (2010) Science, 330, 472-476. [27] Hendrix (2020) Decadal White Paper. [28] Moriarty (2020) Lunar Surface Sci. Wkshp., Abs\# 2241. [29] Hörz et al. (1971) JGR, 76(23), 5770-5798. [30] Mainzer (2020) 23 SBAG Meeting. [31] Mainzer (2020) Decadal White Paper. 